\title{
Enhancing Traffic Performance of Scale-free Networks by Employing Hybrid-information-based Queuing Strategy
}

\author{
Yulin Lu, a, Fenlin Liu², b \\ ${ }^{1}$ State Key Laboratory of Mathematical Engineering and Advanced Computing, Zhengzhou \\ 450001, China. \\ 2 Zhengzhou Science and Technology Institute, Zhengzhou 450001, China. \\ a luyulin_spike@163.com, bliufenlin @vip.sina.com
}

Keywords: Scale-free network, queuing strategy, networked traffic system.

\begin{abstract}
Traffic congestion problem has become a bottleneck restricting the development of modern transportation systems, and accordingly the networked traffic system has drawn lots of researcher's attention. To enhance transportation efficiency on scale-free networks, we proposed a hybridinformation-based (HIB) queuing strategy, where one packet's priority is related to its available transmission resource in the surplus path and current distance to destination. Numerical results show that the HIB queuing strategy perform much better than the traditional first-in-first-out (FIFO) queuing strategy in the congestion state, and the average travelling time, the rate of waiting time to travelling time $\mathrm{P}_{\mathrm{wt}}$ and the packet arrived rate which can reflect transportation efficiency are improved remarkably. Our work should be valuable and helpful for research on networked traffic systems.
\end{abstract}

\section{Introduction}

Ever since the small-world [1] phenomenon and scale-free properties [2] were identified, complex networks have been applied to describe a wide range of natural and society systems in which entities are connected by physical or abstract links. Research has been focused on topologies of networks as well as dynamics of information packets [3-5], spreading of epidemics [6-8], cooperative behaviors of evolutionary games [9-11], and so on. Due to the importance of the large networked systems, many researches have been focused on ensuring free traffic flow and avoiding traffic congestion on complex networks. It has been widely revealed that both the network topology and the routing protocol could affect transport efficiency of the networked system. Thus, there are two general ways to raise the critical packet generation rate where the phase transition takes place: to optimize the underlying network topology $[12,13]$ and to develop new routing protocols [14-16].

To our knowledge, the delivering capacity of nodes is finite in general. If the queuing length is larger than one node's delivering capacity, the packets are temporarily stored in the buffer. There can be different queuing strategies that control the order of packet delivering in the queue buffer. The most common method is the "first-in-first-out" (FIFO) queuing strategy, and the priority queuing strategy is also adopted in previous works $[17,18]$. Kim et al. studied the packet transport problem on scale-free networks under the priority queuing strategy, and they found that the transportation efficiency can be improved in the congestion state, but it is worse in the free-flow state and the jamming transition point is reduced [17]. Du et al. introduce a shortest-remaining-path-first (SRPF) queuing strategy into a network traffic model on scale-free networks under efficient routing protocol, the traffic performance is improved in the congestion state and it will not harm the traffic performance in the free-flow state [18]. Inspired by their works, we proposed a hybrid-information-based (HIB) queuing strategy in which a packet's priority is determined by its available transmission resource in the surplus path and the distance from its current location to destination. It is found that, compared with FIFO queuing strategy, the network capacity has no obvious changes, but some indexes reflecting transportation efficiency can be improved remarkably by the HIB queuing strategy.

This paper is organized as follows. In the following section, the network traffic model is described in detail. The simulation results and discussions are given in Section 3. The paper is concluded by the last section. 


\section{The Networks Structure and Traffic Model}

Empirical studies revealed that realistic networks are heterogeneous with power law degree distribution, here we use the well-known Barabási Albert (BA) model [2] to represent the structure of traffic systems. Most previous studies consider all nodes in networks with homogeneous delivering capacity. But hub nodes usually need to handle more traffic load than others in real-world situation and therefore they are assigned with larger delivering capacity. To capture this fact, the delivering capacity is often assumed to be $\lambda k_{i}$ for a node with degree $k_{i}[19,20]$, where $\lambda \geq 0$ is an adjustable parameter. Without loss of generality, we set $\lambda=1$ in this work and then the delivering capacity of node $\mathrm{i}$ (denoted as $C_{i}$ ) becomes $C_{i}=k_{i}$. Then the whole traffic process can be described as follows: all nodes in the network are equally considered as hosts and routers to generate and deliver packets. At each time step, the network creates $\mathrm{R}$ new packets with randomly chosen sources and destinations. For each packet, once its source and destination are fixed, a path from source to destination is calculated based on the specific routing protocol. In our work, we adopt the shortest path routing protocol. Each node can deliver at most $C_{i}$ packets towards their destinations in one-time step. If it has more than $C_{i}$ packets waiting in its queue at node i, only the first $C_{i}$ packets can be delivered and the rest must wait in the queue buffer according to a certain queuing strategy. Each node is allowed to have an infinite queue length.

In this paper, we propose a hybrid-information-based queuing strategy which employs a priority index $P$ for each packet wait in the queue buffer. The priority index $P$ for a packet $\mathrm{x}$ of node $\mathrm{i}$ at time $t$ can be denoted as

$$
P_{x}^{i}=\left[\sum_{i \in p_{x}} \frac{L_{i}(t)}{C_{i}}\right]^{\alpha} \times D_{r}^{1-\alpha}
$$

where $p_{x}$ is the path of packet $\mathrm{x}$ from node $\mathrm{i}$ to its destination, $L_{i}(t)$ is the queue length at node $\mathrm{i}$ at time $\mathrm{t}, \sum_{i \in P_{x}} \frac{L_{i}(t)}{C_{i}}$ denotes the available transmission resource in the surplus path of packet $\mathrm{x}$ at time $\mathrm{t}$, $D_{r}$ is the distance from current location to destination of packet $\mathrm{x}$ at time $\mathrm{t}$, and $\alpha$ is a tunable parameter with a value ranging from 0 to 1 . Packets in the queue buffer will be delivered in descending order according to the value of priority index. In all the present simulations, the network size is $\mathrm{N}=500$, the average degree is $<\mathrm{k}>=4$, and the total simulation time step is $\mathrm{T}=10000$.

\section{Literature References Discussion of Simulation Results}

In this section, we compare the HIB queuing strategy with the traditional FIFO queuing strategy to investigate the traffic behavior on BA scale-free network model. In order to find the overall traffic capacity, we first investigate the order parameter introduced in [21], which reads

$$
\eta(R)=\lim _{t \rightarrow \infty} \frac{C}{R} \frac{\left\langle\Delta N_{p}\right\rangle}{\Delta t}
$$

where $\Delta N_{p}=N_{p}(t+\Delta t)-N_{p}(t),<\cdots>$ indicates the average over time windows of width $\Delta \mathrm{t}$, $\mathrm{C}$ is the average delivering capacity over all nodes, and $N_{p}(t)$ denotes the number of packets in the network at time $t$. With increasing packet generation $\mathrm{R}$, there will be a critical value of which characterizes the phase transition from free-flow state to congestion state. In free-flow state, $\eta(R)$ is around zero due to the balance of created and removed packets. When $\mathrm{R}$ exceeds $R_{c}$, the packets accumulate in the network, and $\eta(\mathrm{R})$ becomes positive and grows with R. From inspection of Fig. 1, one can see that HIB queuing strategy at different and FIFO queuing strategy undergoes a continuous transition at the same value of R. Our HIB queuing strategy does not affect the value of $R_{c}$ since the packet's routing path is not modified. However, the order parameter $\eta(R)$ under the HIB queuing 
strategy is remarkably lower than that of FIFO queuing strategy in the congestion state, especially at higher packet generating rate. Because the value of $\eta(R)$ can accurately describe the accumulation of packets in the network, lower order parameter obtained by our method indicates that the HIB queuing strategy can improve the traffic performance in the congestion state compared with FIFO. The performance of our proposed HIB queuing strategy depends on the parameter $\alpha$, the order parameter $\eta(R)$ has the minimal value over the whole given range of packet generating rate $\mathrm{R}$ when we set $\alpha=$ 0.8 . Therefore, we focus our attention to the traffic performance obtained at the optimal parameter $\alpha=$ 0.8 .

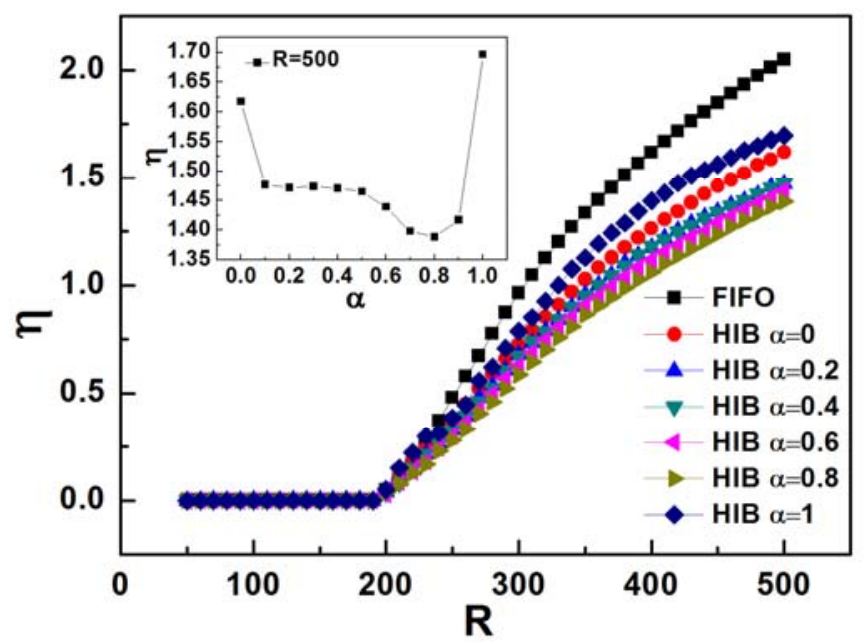

Fig 1. The Relationship of the Order Parameter $\eta(R)$ and the Packet Generating Rate $R$ under the HIB Strategy with Different Parameter $\alpha$.

The average travelling time of arrived packets $<\mathrm{T}>$, which can reflect the traffic efficiency, is a critical index for traffic systems. From the inspection of Fig. 2(a), one can see that $<\mathrm{T}>$ of HIB and FIFO queuing strategies keeps the same value in the free-flow state, but the HIB queuing strategy is dramatically smaller than the FIFO queuing strategy in the congestion state. In particular, our HIB queuing strategy has superiority in severe congestion state over the FIFO queuing strategy. At the packet generating rate $\mathrm{R}=500,<\mathrm{T}>$ is 1,982 for the FIFO queuing strategy, while it is only 86 for the HIB queuing strategy for $\alpha=0.8$. The rate of waiting time to travelling time $P_{w t}$ is an index which can represents the user's satisfaction of traffic system. For example, in many networked systems, especially for urban traffic systems, passengers will be intolerable if $P_{w t}$ is too large. Therefore, the less $P_{w t}$ is, the higher the users satisfaction is. It can be denoted as

$$
P_{w t}=\left(\sum_{i=1}^{N_{\text {arrived }}} T_{i-\text { wait }} / T_{i-\text { travel }}\right) / N_{\text {arrived }}
$$

Where $T_{i \text {-travel }}$ the total travelling is time of packet $\mathrm{i}, T_{i \text {-wait }}$ is the total waiting time of packet $\mathrm{i}$, and $N_{\text {arrived }}$ is the number of arrived packets. From observation of Fig. 2(b), one can see that the HIB queuing strategy evidently outperforms the FIFO queuing strategy. The $P_{w t}$ of the HIB queuing strategy keeps steadily much lower than the FIFO queuing strategy over the whole given range of the congestion state from the critical rate $R_{c}$ to $\mathrm{R}=500$. At $\mathrm{R}=500$ and $\alpha=0.8, P_{w t}$ of the HIB queuing strategy was reduced by $77 \%$ compared with that of the FIFO queuing strategy. Then we turn to result of the packet arrived rate under HIB queuing strategy. The packet arrived rate is the index denoting system throughput, which indicates the delivery capacity of the whole network. The packet arrived rate $\mathrm{Q}$ is defined as 


$$
Q=\frac{N_{\text {arrived }}}{N_{\text {total }}}
$$

The relationship between $\mathrm{Q}$ and $\mathrm{R}$ is shown in Fig. 2(c). In the free-flow state, almost all packets can successfully arrive at their destinations and thus Q is close to 1 . However, in the congestion state, not all packets can arrive at their destinations and thus $0<\mathrm{Q}<1$. Compared with the FIFO queuing strategy, the HIB queuing strategy achieved higher values of $Q$ with no exception. In summary, the traffic efficiency is greatly improved over the FIFO queuing strategy by employing our proposed HIB queuing strategy. One can find that the HIB queuing strategy formalism at $\alpha=0.8$ has the best performance of traffic efficiency for all the three characteristic quantities.

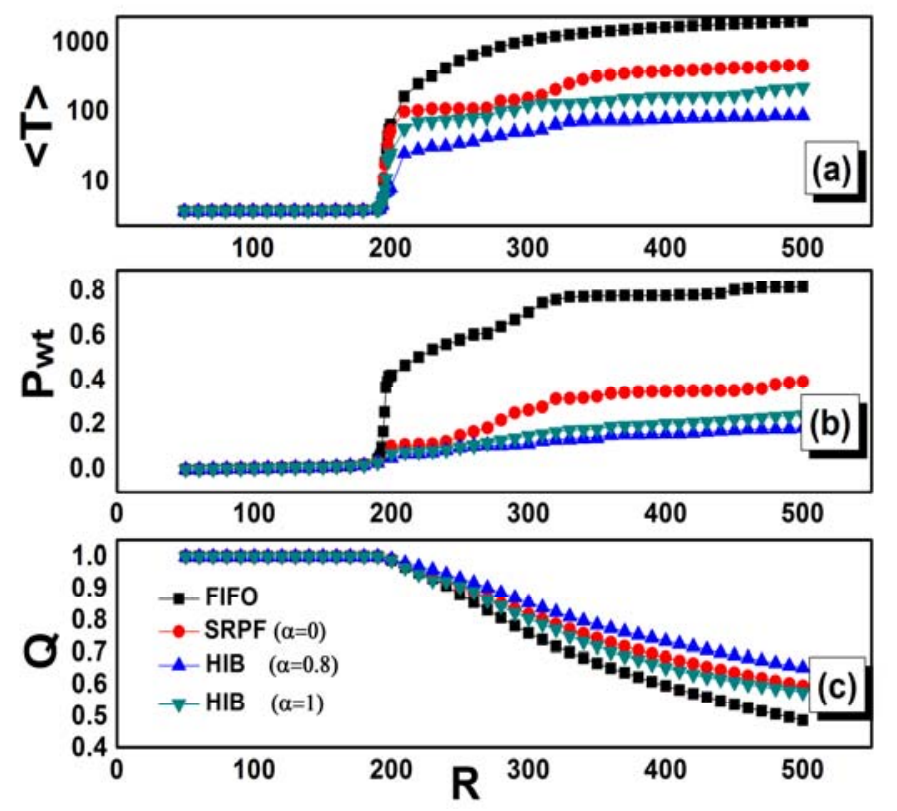

Fig 2. The Functional Relations with the Packet Generating Rate R of (a) the Average Traveling Time $<\mathrm{T}>$, (b) the Rate of Waiting Time to Travelling Time $P_{w t}$, and (c) the Packet Arrived Rate.

From discussions above, one can find that the several critical indexes reflecting transportation efficiency are greatly improved in the congestion state under the HIB queuing strategy. In the following we investigate the mechanism of HIB queuing strategy enhancing the traffic performance. The queuing strategy can only adjust the delivering order of packets in queue buffer and thus there are two ways to improve the traffic performance. One is let some packets get to the destination as soon as possible so that they will be removed from the system and hence the total traffic load is alleviated. The authors of Ref. [18] considered the packet shortest-path-length-remained information when proposing their queuing strategy based on this standpoint, and the traffic performance was improved while it is unfair for long-path packets taking much longer travelling time. Another way is to delay the packets delivering to the congested nodes, so that the traffic load between congested nodes and uncongested nodes can be balanced. Thus, we employ the packet's available transmission resource in the surplus path to capture this fact. We first investigate the load distribution and packet arrived rate of various path length when only the packet's available transmission resource information works in the HIB queuing strategy $(\alpha=1)$. One can see from Fig. 3(a), the traffic load of hub nodes under the HIB queuing strategy is much lighter than that under the FIFO queuing strategy, while it is slightly heavier at the non-hub nodes than that under the FIFO queuing strategy. That indicates the information of packet's available transmission resource in the surplus path can ensure the queuing strategy balance the traffic load between congested nodes and uncongested nodes. However, one can see from Fig. 3(b) that the packet's arrived rate of short path length packets for HIB queuing strategy is slightly smaller than the FIFO queuing strategy when $\alpha=1$. Since the short path length packets are in the majority of the system, it is harmful and unfair for the low packet's arrived rate of short path length packets. Noticeably, the HIB strategy degenerates to the SPRF strategy recently proposed 
in Ref. [18] when $\alpha=0$. To summarize, there are obvious shortcomings for designing queuing strategy only consider the information of packet's available transmission resource in the surplus path or the distance from current location to destination separately. The results in Fig. 3(c) and Fig. 3(d) show the load distribution and packet arrived rate of various path length for the HIB queuing strategy via tunable parameter $\alpha=0.8$. We find that the traffic load of hub nodes under the HIB queuing strategy is lighter than that under FIFO queuing strategy, and the packet arrived rate of various path length is also larger than that of FIFO queuing strategy. The simulation results show that the HIB queuing strategy not only can make short path packets get their destination quickly, but also can balance the traffic load between congested nodes and uncongested nodes. Moreover, we have shown that the HIB queuing strategy combined the hybrid information effectively with appropriate selection of the tunable parameter.
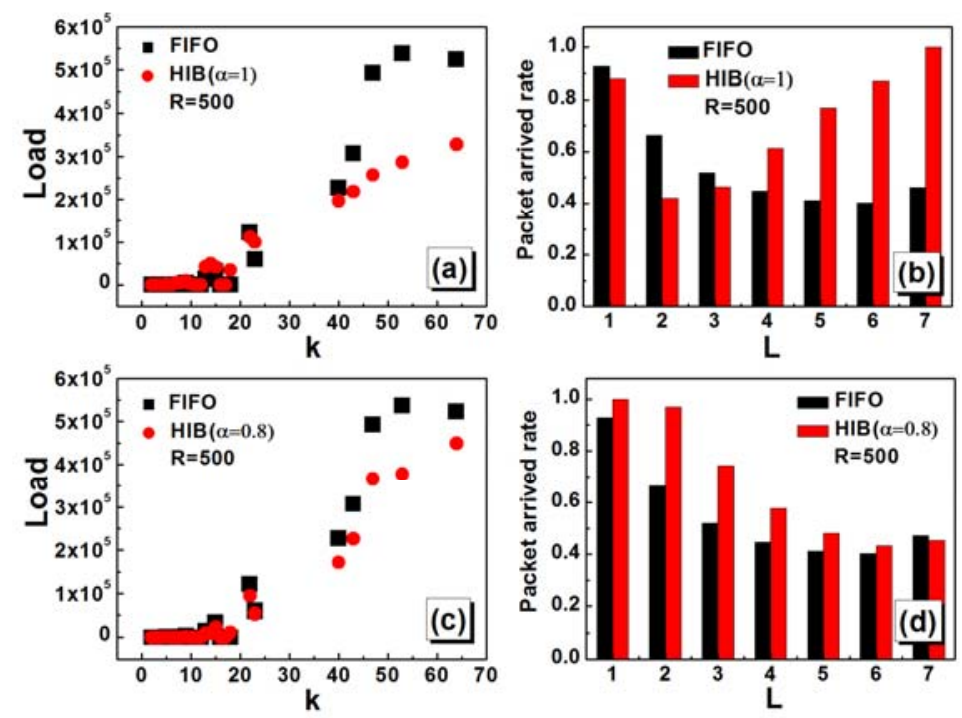

Fig 3. The Load Distribution and the Packet Arrived Rate for Various Path Length L in the Congestion State under Two Queuing Strategies.

\section{Summary}

In summary, we proposed a hybrid-information-based queuing strategy (HIB) which makes packets in the queue buffer to be delivered in descending order according to the value of priority index. Through numerical simulations, the HIB queuing strategy has proved its superior traffic efficiency compared with the traditional FIFO queuing strategy and the SRPF queuing strategy recently proposed by $\mathrm{Du}$ et al. [18]. The performance of the average travelling time $\langle\mathrm{T}\rangle$, the rate of waiting time to travelling time $P_{w t}$ and the packet arrived rate $\mathrm{Q}$ are dramatically improved in the congestion state. By adjusting the tunable parameter $\alpha$, networked traffic system under the HIB queuing strategy can reach the optimal traffic performance. The optimal traffic performance is found at parameter $\alpha=0.8$. Our work may provide novel insights for research on networked traffic systems.

\section{References}

[1]. WattsD J, Strogatz S H. “Collective dynamics of 'small-world' networks," Nature. Vol. 393 (1998) No. 6684, p. 440-442.

[2]. Barabási A L, Albert R. "Emergence of scaling in random networks," Science. Vol. 286 (1999) No. 5439, p. 509-512.

[3]. Yan G, Zhou T, Hu B, et al. "Efficient routing on complex networks," Physical Review E. Vol. 73 (2006) No. 4, p. 046108. 
[4]. Wang W X, Wang B H, Yin C Y, et al. "Traffic dynamics based on local routing protocol on a scale-free network," Physical Review E. Vol. 73 (2006) No. 2, p. 02611.

[5]. Hu M B, Ling X, Jiang R, et al. "Dynamical hysteresis phenomena in complex network traffic," Physical Review E. Vol. 79 (2006) No. 4, p. 047101.

[6]. Pastor-Satorras R, Vespignani A. "Epidemic spreading in scale-free networks," Physical review letters. Vol. 86 (2001) No. 14, p. 3200.

[7]. Zhao M, Zhou T, Wang B H, et al. "Relations between average distance, heterogeneity and network synchronizability," Physica A: Statistical Mechanics and its Applications. Vol. 371 (2006) No. 2, p. 773-780.

[8]. Yang H X, Wang W X, Lai Y C, et al. "Control of epidemic spreading on complex networks by local traffic dynamics," Physical Review E. Vol. 84 (2011) No. 4, p. 045101.

[9]. Szabó G, Fath G. "Evolutionary games on graphs,” Physics Reports. Vol. 446 (2007) No. 4, p. 97-216.

[10]. Roca C P, Cuesta J A, Sánchez A. "Evolutionary game theory: Temporal and spatial effects beyond replicator dynamics," Physics of Life Reviews. Vol. 6 (2009) No. 4, p. 208-249.

[11]. Perc M, Szolnoki A. "Coevolutionary games - a mini review," BioSystems. Vol. 99 (2010) No. 2, p. 109-125.

[12]. Huang W, Chow T W S. "An efficient strategy for enhancing traffic capacity by removing links in scale-free networks," Journal of Statistical Mechanics: Theory and Experiment. (2010) No. 01, p. 01016.

[13]. Jiang Z, Liang M, Guo D. "Enhancing network performance by edge addition," International Journal of Modern Physics C. Vol. 22 (2011) No. 11, p. 1211-1226.

[14]. Xi Z, Zhou Z, Dong C. "Efficient path routing strategy for flows with multiple priorities on scale-free networks," Plos One. Vol. 12 (2017) No. 2, p. e0172035.

[15]. Jiang Z Y, Ma J F, Jing X. "Enhancing traffic capacity of scale-free networks by employing hybrid routing strategy," Physica a Statistical Mechanics \& Its Applications. Vol. 422 (2015) p. 181-186.

[16]. Hong C. "Effective usage of global dynamic information for network traffic," Physica A Statistical Mechanics \& Its Applications. Vol. 424(2015) p. 242-247.

[17]. Kim K, Kahng B, Kim D. "Jamming transition in traffic flow under the priority queuing protocol,” EPL (Europhysics Letters). Vol. 86 (2009) No. 5, p. 58002.

[18]. Du W B, Wu Z X, Cai K Q. "Effective usage of shortest paths promotes transportation efficiency on scale-free networks," Physica A: Statistical Mechanics and its Applications. Vol. 392 (2013) No. 17, p. 3505-3512.

[19]. Liu Z, Ma W, Zhang H, et al. "An efficient approach of controlling traffic congestion in scalefree networks," Physica A: Statistical Mechanics and its Applications. Vol. 370 (2006) No. 2, p. 843-853.

[20]. Tang M, Liu Z, Liang X, et al. "Self-adjusting routing schemes for time-varying traffic in scale-free networks," Physical Review E. Vol. 80 (2009) No. 2, p. 026114.

[21]. Arenas A, Díaz-Guilera A, Guimera R. "Communication in networks with hierarchical branching," Physical Review Letters. Vol. 86 (2001) No. 14, p. 3196. 